\title{
The Relationship Between Drug Addiction and Adolescent Cognitive Development
}

\author{
${ }^{1}$ Suzhou North American High School, Suzhou, Jiangsu, China \\ ${ }^{2}$ School of McMaster University, Hamilton, ON, CA \\ *Corresponding author. Email: zhour26@mcmaster.ca \\ Those authors contributed equally.
}

Shengliang Shau ${ }^{1, \dagger}$, Ruiao $\mathrm{Zhou}^{2, *}, \dagger$

\begin{abstract}
As drug abuse among adolescents is becoming a serious social concern, this article is aiming at reviewing the negative impacts that drugs can bring to adolescents' brain development, finding out what are the causes for adolescents getting attachments to the drugs, and what are the methods for avoidance and treatments. In the existing evidence, the drug's impact on brain development is clear, but the details for the damages did not come to an agreement. The main influence of consuming drugs on brain development is on cognitive and memory levels, and the accesses are mostly found in peer groups, households, and school places. In this review study, the evidence showed the positive results of drug addiction recovery in Behavioral Therapies and Family Therapies. This review study will be a quick guide for knowing the adolescent drug use phenomenon and its available treatment methods.
\end{abstract}

Keywords: drug abuse, substance usage, adolescents, therapies.

\section{INTRODUCTION}

Drug abuse is a serious problem today, and this behavior is not well controlled. Many drugs can alter a person's thinking and judgment and can lead to health risks, including addiction, drugged driving, infectious disease, and adverse effects on his, her, their, etc. pregnancy [1]. Very common drugs include alcohol, cocaine, heroin, methamphetamine, and tobacco/nicotine. Adolescents are at high risk of drug abuse and addiction, and what can different kinds of drugs affect their cognitive development, and what might be the consequences remain unclear. Since marijuana became legal in New York state, there is an escalated number of sales in New York state. According to the health department of New York City, most of the consumption of marijuana is adolescents [2]. However, adolescents have limited knowledge of the negative effects. According to Leshner [3], drug addiction could be regarded as a brain disease since it is tied to changes in brain structure and function. Different drugs affect the brain differently, and drug abuse can lead to serious consequences. Heroin is converted to morphine in the body. It binds to opioid receptors in the brainstem. Opioid receptors inhibit neuron activities. Overdoses can cause hard breathing [4]. Methamphetamine is one of the hallucinations. It increases norepinephrine, dopamine, and serotonin. Overdoses can decrease salivation; increase dehydration, teeth grinding, and general lack of concern for hygiene. There are some other impacts such as sweating, racing heart, vomiting, anxiety, insomnia, social isolation, etc. One research claims that using marijuana shows a decline in neuropsychological decline [5]. However, another research shows that marijuana use does not have significant effects on the intelligence quotient [6]. Therefore, some conflicts still exist in the exact effect of drug abuse on individuals 'cognitive functions.

Furthermore, a lot of evidence showed that adolescence is a period where our brain and cognitive ability develop fast, in a recent structural MRI study have demonstrated that the brain undergoes considerable development during adolescence, both our crosssectional and longitudinal data demonstrate that changes in the frontal and parietal regions are especially pronounced and prolonged [6]. Also, in this stage of adolescence, the risk-taking behaviour is increasing, the willingness to try every possibility for themselves tends to become a need. To have a clear picture of what many different types of drugs can cause to adolescents and understand the methods to limit adolescents 'drug use behaviour is extremely important. The focus of this paper is on providing a better understanding of drugs and drug 
addiction to the public. Among many of the studies based on the drug abuse problem, what are the causes that make adolescents at high risk of adapting drug abuse behaviours since this behaviour may lead to serious consequences becoming emerging. To be clear of what are the negative outcomes can drug abuse brings to adolescents is another goal of this review, the negative effects cannot only be on a physical level, but also on the social perspective, which includes the drug effects on personal health, cognitive functioning abilities, more risk-taking behaviours and the negative effects on social functioning and its impact to other members of the society. This review will also compare different methods of therapies to find out what are the possible solutions, and by taking what kind of actions may prevent adolescents 'drug use behaviours. The articles and papers 'limitations and their further implications will also be discussed by the end of this review and seeking out the gaps between different studies will be a final part of this review's aim.

\section{THE CAUSES OF ADOLESCENTS' DRUG ABUSE}

\subsection{Peer Influence}

Peer is a great part of the influential factor for adolescents, as adolescents try on new identities, assume more responsibility, and develop views distinct from those of their parents, they place greater value on their peers 'opinions [7]. Peers provide affection, fun, and act as buffers from difficult relationships at home or in school, and it has been shown that adolescents with strong friendships are more confident, altruistic, less aggressive, better adjusted with enhanced social skills and fewer internalizing problems and demonstrate greater school involvement and work orientation than same-aged peers without these relationships [7]. Consequently, peer interactions become highly essential for teenagers, perhaps more so than parent-child ties in certain cases. Communication has become even simpler for teenagers to contact their classmates in modern times, thanks to the rapid growth of social media and the fact that nearly every adolescent has access to the internet. In addition, adolescents 'time on using social media keeps increasing, which means their interaction with their peers becomes more frequent. However, there are many negative sides that peers could bring to adolescents, one common example is peer pressure. Peer group has a high influence on the decision-making process of adolescents, if the adolescent's peers are involving in drug use behaviours, it would be hard for the individual to refuse, sometimes the drug use behaviour may even be a decision the adolescent's peer group help to make for that individual, rejection of adapting that made decision may lead to isolation. Previous study showed peers are the largest influence of direct drug use to adolescents, adolescents at higher stages of drug use had parental models of drug use and either peer who were negative and rejecting or peer models of drug use [7]. Adolescent's fear losing their peers, in fact, losing a peer relationship may cause internalizing problems including depression, anxiety, loneliness, and poor self-esteem. Adolescents with a peer group who have problems involving drug use are at high risk of drug addiction and losing their peers may also result in behaviours like drug use.

\subsection{Family/Background}

There are many factors that can lead adolescents to drug addiction, environment, and family background (genetics/family influence) are two major factors that contribute to drug abuse [1]. According to the National Council in Alcohol and Drug Dependence National Council on Alcoholism and Drug Dependence of the Central, a person whose family has a history of substance abuse is twice as likely to develop mental health issues and substance abuse problems themselves. From the adolescent drug addiction perspective, drug addiction can be both nature and nurture. For instance, if the adolescent's family has a history of drug abuse, there is a high possibility of adapting to this behaviour. Investigations have shown that addiction tends to be heritable, depending on the individual's relationship to the person with the substance addiction [1]. To put it another way, if one or more of a teenager's first-degree relatives have a substance use problem, the adolescent is at a high risk of being addicted to alcohol or drugs. Low self-esteem, scholastic issues, loneliness, melancholy, and the need for social acceptability are all risk factors for teenage drug usage, according to a study from Columbia University's National Center on Addiction and Substance Abuse. Families are having negative communication, which ignores adolescents 'needs, with a lot of criticism, complaints often are triggering adolescents to develop substance abuse due to stress, and factors such as inconsistent parenting, unrealistic expectations, denial, misplaced anger, and selfmedication may also result correspondingly [1].

\subsection{Access to Drugs}

Numerous adolescents are involving infrequent use of drugs, according to the statistics in $2017,29 \%$ of high schoolers regularly use alcohol, $12.5 \%$ of high school students regularly binge drinking; $19.8 \%$ of high schoolers regularly use marijuana; $7.5 \%$ of 12 th graders have vaped marijuana in the past month; $12.4 \%$ of 12 th graders have used illicit drugs (other than marijuana) in the past year; $11 \%$ of high school seniors have misused prescription medications in the past year; $7.9 \%$ high school seniors regularly misuse amphetamines, such as Adderall or Ritalin; and 4\% of high schoolers regularly misuse pain medications [8]. The question here is, where do adolescents get their drugs? In fact, adolescents have 
many accesses to 'buy'drugs without buying drugs from stores. One commonplace that the drugs are being transacted is in the school place, nearly $20 \%$ of high school students claim they had been offered drugs on school property, and most often, their peers are the ones who provide the drugs. Since adolescents are under the legitimate age for consuming drugs from stores, a fake id is a perfect solution. There are proven cases that there are fake ID businesses in North America, they help adolescents to create their fake IDs such as drivers' licences. Besides that, some adolescents might use other's IDs of those who look like them. Adolescents may also take drugs at home which belong to their parents, substances like alcohol and cigarettes can be easily found in many households, and the drugs adolescents find in their home may be brought to school and sold/share with their peers. Shopping for drugs online becomes a new method for adolescents to get access to drugs. As social media and online shopping become easier than ever, adolescents can find their access to drugs by online shopping, they can either shop from online pharmacies without a clear prescription, or from illegitimate online pharmacies.

\section{IMPACTS OF ADOLESCENT DRUG USAGE}

Drug use is very common among teenagers, and a few of the popular drugs are marijuana and cocaine. Different drugs would have different impacts cognitively, in the short term and the long term. Substance usage can be very harmful even for adults, adolescent's developing brains would increase the harm brought by drugs causing life-long damages.

\subsection{Cognitive Consequences due to Marijuana usage}

Squeglia et al. explained how to find people who regularly smoke marijuana through lower performance tests to measure learning, cognitive flexibility, working memory [9]. Multiple experiments and studies were done and analyzed within the study, to examine the different effects caused by adolescent substance use within the brain structure and functions. Part of the results showed how substance usage would influence adolescents 'brain structure, in terms of their prefrontal cortex volume, gray matter volume, and white matter volume. On average, female cannabis users exhibited larger posterior and prefrontal cortex volume than non-smokers, which is thought to be related to poor verbal memory and therefore suggests that synaptic pruning may be disrupted. Moreover, an increase in marijuana usage among adolescents showed a larger global gray matter volume compared to the controlled groups, suggesting a disruption of the gray matter pruning process, along with a predicted result of having poorer verbal and attention performance. The teenage marijuana users showed relatively poor performance, particularly in the learning trials, on the "extensive neuropsychological test battery" -including verbal learning and memory tests.

\subsection{Cognitive Consequences due to Cocaine exposure}

Cocaine is one of the most widely used drugs other than marijuana among adolescence that would also influence their under-developed brain in several ways. Results from [9] showed that Rats exposed to cocaine during adolescence have poor ability to develop their situational fear responses. Other studies have concluded how early exposure to cocaine will affect the mechanisms that regulate nutritional responses [10]. Furthermore, Cocaine use that is chronic and repetitive might cause neuroplasticity impairment [11]. According to a review by Caffino et al. [12], the adolescent's response to the first cocaine injection would predict his or her response to the second. Furthermore, based on this finding it also suggests that "responses to a subsequent event later in life" might be influenced by the exposure to cocaine during adolescence. Experiments done on adolescent rats, to simulate teenagers 'reactions to cocaine, have suggested that depressive-like status may occur with a single injection of cocaine, due to the underdeveloped brain. Based on these findings, predicting the sensitivity of the motivational systems may be possible for the following exposure to cocaine, if "the neuroadaptive changes set in motion" by the first exposure are understood. Adolescents 'brain development aren't the only victims of teenage drug abuse, the people around the patient would also be influenced.

\section{BEHAVIOURAL THERAPIES}

\subsection{Contingency Management Therapies}

According to Petry [13], Contingency Management Therapies (CMT) is a type of highly effective behavioural treatment for substance usage; the main idea of CMT is to encourage patients to make positive behavioural changes, by motivating them through positive incentives such as rewards. The CMT is based on the principles of basic behavioural analysis [13], explaining how behaviours with positive consequences are more likely to be repeated or done instead of ones with negative consequences [14]. For example, there are two approaches to reducing illicit drug use: one enables patients to take home methadone doses (medications used to treat withdrawal symptoms) if they agree to produce drug-free urine samples, while the other decreases methadone doses if the requirements are not fulfilled). [14]. According to the principles of basic behavioural analysis, the method that provided positive consequences (taking methadone doses home) would 
bring a comparatively significant reduction in illicit drug use [14].

Before the 1990s, it was difficult for the CMT to apply in circumstances other than the methadone maintenance programs [14], Until Dr. Higgins and his colleagues demonstrated the efficacy of voucher-based reinforcement treatment, it was mostly used to treat cocaine addiction [15]. Patients (cocaine addicts) would undergo three urine specimen tests each week as part of Dr. Higgin's therapy, and if all three tests were negative for cocaine, the patients would be awarded recorded vouchers that could be exchanged for things that encourage healthy living. [15]. The results of the experiments showed how the voucher treatment groups would have much higher rates in completely the 12 or 24 weeks treatment, while the average cocaine abstinence from voucher groups -11.7 weeks- are almost twice if the non-voucher groups -6 weeks- [15].

\subsection{Cognitive Behaviour Therapy}

Unlike other therapies, cognitive behavioural therapy (CBT) focuses on altering one's thoughts rather than changing one's surroundings or using rewards to drive one's behaviour. CBT is founded on the idea that "ideas create feelings and behaviours," and that patients may reduce their substance use by learning, comprehending, and accepting the reasons for their thoughts or actions that lead to substance use [16]. Other than using CBT as a treatment to reduce substance use, it can be used as a treatment for mental disorders like depression, anxiety, and such [16]. Two of the major features of cognitivebehavioural approaches are functional analysis and skill training [15]. Functional analysis is a type of cognitive behavioural therapy (CBT) in which patients learn about their cause or reason for substance abuse. Specific questions may be used to assist patients to recollect their previous experiences prior to using the drug [16]. The term skill training, learning self-management skills or coping skills while recognizing situations that one is most vulnerable to drug use and avoiding the situations [15].

In the long term, CBT is a more successful therapy than the other therapies. According to studies, the effects of CBT are generally long-lasting, and improvements in drug use are more likely to occur even after therapy.

On drug users, one of the research evaluated the effectiveness of CMT with CBT. Although the results revealed that CMT was more successful than $\mathrm{CBT}$, the long-term results showed that patients allocated to the CBT group exhibited a substantial rise in improvements even after a year, compared to "weakening effects for contingency management." [15]. Therefore, the contingency management method is more effective for short-run treatments, and cognitive behaviour therapy in the long run.

\subsection{Family Therapies}

Family Therapy has been generally defined as a therapy that emphasizes the role of the family [17], or strengthening the interaction between family members to resolve one's problem — can be used for reducing substance (usually alcohol or drug) usage [18]. Throughout the treatment, family members and the drug user would learn about the reason of action, methods to enhance communication between family members, addressing family roles, and other topics according to their circumstances [18]. There are still many distinct forms of family therapy, such as structural family therapy, conjoint family therapy, one-person family therapy, improved engagement programs, and so on. The duration, tactics, and procedures of the various family treatments vary [19]. An example of structural family therapy is the first successful case to reduce adolescents 'drug usage from Szapoczink and his colleagues in the 1960s, starting with the abstinence rate of $7 \%$ and ending with a rate of $80 \%$ [19]. Not only, their study has shown not only how successful structural family therapy is in reducing teenage drug use, but also how cultural factors may be used in clinical research.). Despite these advantages, the limits of family therapy should be recognized; a very limited number of researchers have studied family therapy compared to all drug consumption studies, thus the effectiveness of family therapy has yet to be verified, leading to uncertainty [19]. However, other than the relatively small number of studies, the results shown by current studies seem promising.

\section{CONCLUSION}

Drug use behaviour by adolescents is a serious social concern based on its negative impact on both social function and individual brain development. According to the research and reviews, peer groups and family background often greatly influence adolescents' behaviour, since a peer group may mislead adolescents ' attitudes and discussion making towards drug consumption, and evidence also showed families with drug abuse backgrounds can highly increase the chance for adolescents to be addicted to drugs. With the finding resources of locations that adolescents are getting their drugs, they are often easy for them to access, the control based on the knowing places should get people's attention. The commonly used drugs among teenagers can be very harmful effects on their cognitive development as explained above. Adolescents 'immature brain structure is one of the major factors that make drug use even more dangerous; irreversible impacts can occur with just a single exposure, and the damages done to the brain would stick with them until adulthood. Repetitive drug usage increases the risks and damages, so certain treatments should be done to the ones already in drug abuse preventing further damage. Given these therapies and their effectiveness, adolescents should be 
encouraged more often to decrease their reliance or even live off substances. Stopping the repetitive use of drugs can be considered the most direct way to reduce the harms brought by it. However, compared to treatments after drug use, preventing drug exposure to adolescents would remain the best solution.

\section{REFERENCES}

[1] Commonly Used Drugs Charts. (2020, August 20). National Institute on Drug Abuse. https:/www.drugabuse.gov/drugtopics/commonly-used-drugs-charts

[2] Cannabis (marijuana). Cannabis (Marijuana) NYC Health. (n.d.). https:/www1.nyc.gov/site/doh/health/healthtopics/marijuana.page

[3] Leshner, A. I. (1998). Addiction is a brain disease-and it matters. PsycEXTRA Dataset. https://doi.org/10.1037/e564232006-001

[4] NIDA. (2020, May 25). Evidence-Based Approaches to Treating Adolescent Substance Use Disorders. National Institute on Drug Abuse. https://www.drugabuse.gov/publications/principles -adolescent-substance-use-disorder-treatmentresearch-based-guide/evidence-based-approachesto-treating-adolescent-substance-use-disorders

[5] Meier, M. H., Caspi, A., Ambler, A., Harrington, H., Houts, R., Keefe, R. S., McDonald, K., Ward, A., Poulton, R., \& Moffitt, T. E. (2012). Persistent cannabis users show neuropsychological decline from childhood to midlife. Proceedings of the National Academy of Sciences, 109(40). https://doi.org/10.1073/pnas.1206820109

[6] Hall, W., \& Carter, A. (2012). Faculty of 1000 evaluation for persistent cannabis users show neuropsychological decline from childhood to midlife. F1000 - Post-Publication Peer Review of the Biomedical Literature. https://doi.org/10.3410/f.717954083.793459861

[7] Crosnoe, R. (2000). Friendships in childhood and adolescence: The life course and new directions. Social Psychology Quarterly, 63(4), 377. https://doi.org/10.2307/2695847

[8] Renzoni, C. (2021a, August 13). Where Teens Get Illicit Drugs. The Recovery Village Drug and Alcohol Rehab. https://www.therecoveryvillage.com/teenaddiction/drug/how-teens-get-drugs/.

[9] Squeglia, L. M., Jacobus, J., \& Tapert, S. F. (2009, January 1). The Influence of Substance Use on Adolescent Brain Development. SAGE Journals. https://journals.sagepub.com/doi/10.1177/1550059 40904000110

[10] National Institute on Drug Abuse. (2020, May 25). Evidence-based approaches to treating adolescent substance use disorders. National Institute on Drug Abuse. Retrieved October 23, 2021, from https://www.drugabuse.gov/publications/principles -adolescent-substance-use-disorder-treatmentresearch-based-guide/evidence-based-approachesto-treating-adolescent-substance-use-disorders.

[11] Giannotti, G., Caffino, L., Malpighi, C., Melfi, S., Racagni, G., \& Fumagalli, F. (2014, August 16). A single exposure to cocaine during development elicits regionally-selective changes in basal basic Fibroblast Growth Factor (FGF-2) gene expression and alters the trophic response to a second injection. SpringerLink.

https://link.springer.com/article/10.1007/s00213014-3708-

$\mathrm{x}$ ?error $=$ cookies_not_supported\&error $=$ cookies_no $\mathrm{t}$ supported\&code $=9 \mathrm{~b} 50 \mathrm{~b} 0 \mathrm{e} 7-2 \mathrm{bc} 9-4 \mathrm{c} 79-94 \mathrm{~d} 8$ e3613f52492b\&code $=3$ e4fbac $1-13$ bd-4425-9e0a$4791 \mathrm{f} 17 \mathrm{e} 93 \mathrm{c} 0$

[12] Caffino, L., Mottarlini, F., Zita, G., Gawliński, D., Gawlińska, K., Wydra, K., Przegaliński, E., \& Fumagalli, F. (2021, June 8). BPS Publications. British Pharmacological Society | Journals. https://bpspubs.onlinelibrary.wiley.com/doi/10.111 1/bph. 15523

[13] Petry, N. M. (2011, May). Contingency management: what it is and why psychiatrists should want to use it. NCBI. https://www.ncbi.nlm.nih.gov/pmc/articles/PMC30 83448/

[14] Carroll, K. M., \& Onken, L. S. (2005, August 1). Psychiatry Online. The American Journal of Psychiatry.

https://ajp.psychiatryonline.org/action/cookieAbse nt

[15] Mueller, M. D. (1995, October 1). Voucher System Is Effective Tool in Treating Cocaine Abuse. NIDA Archives. https://archives.drugabuse.gov/newsevents/nida-notes/1995/10/voucher-systemeffective-tool-in-treating-cocaine-abuse

[16] Buddy, T. (2020, November 6). Can Cognitive Behavioral Therapy (CBT) Treat Addiction? Verywell Mind. https://www.verywellmind.com/cognitivebehavior-therapy-for-addiction-67893

[17] Renzoni, C. (2021, September 29). Substance Abuse Treatment Options for Teens. The Recovery Village Drug and Alcohol Rehab. 
https://www.therecoveryvillage.com/teenaddiction/drug/substance-abuse-treatment-options/

[18] Dean, M. E. (2021, January 29). Family Therapy Techniques: How Family Counseling Works | BetterHelp.

Betterhelp. https://www.betterhelp.com/advice/family/familytherapy-techniques-how-family-counseling-works/

[19] Liddle, H. A., \& Dakof, G. A. (1995, October). EFFICACY OF FAMILY THERAPY FOR DRUG
ABUSE: PROMISING BUT NOT DEFINITIVE.

Wiley Online Library.
https://onlinelibrary.wiley.com/doi/10.1111/j.1752 -0606.1995.tb00177.x

[20] Choudhury, S., Blakemore, S. J., \& Charman, T. (2006, December 1). Social cognitive development during adolescence. Oxford Academic. https://academic.oup.com/scan/article/1/3/165/236 2733 ? $\log$ in $=$ true 